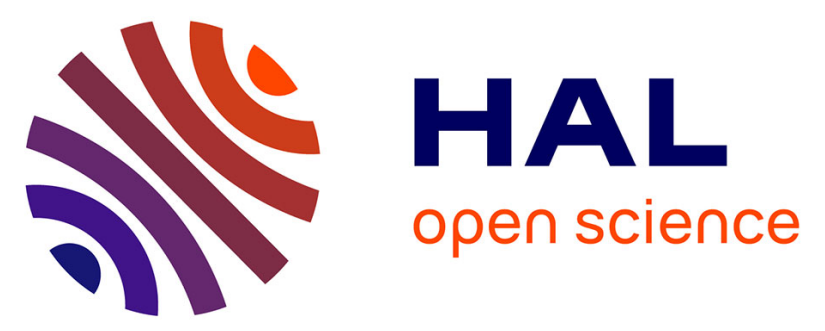

\title{
Microchannel-connected SU-8 honeycombs by single-step projection photolithography for positioning cells on silicon oxide nanopillar arrays
}

Florian Larramendy, Marie-Charline Blatché, Laurent Mazenq, Adrian Laborde, Pierre Temple-Boyer, Oliver Paul

\section{To cite this version:}

Florian Larramendy, Marie-Charline Blatché, Laurent Mazenq, Adrian Laborde, Pierre Temple-Boyer, et al.. Microchannel-connected SU-8 honeycombs by single-step projection photolithography for positioning cells on silicon oxide nanopillar arrays. Journal of Micromechanics and Microengineering, 2015, 25 (4), pp.045010. 10.1088/0960-1317/25/4/045010 . hal-01225646

\section{HAL Id: hal-01225646 \\ https://hal.science/hal-01225646}

Submitted on 10 Nov 2015

HAL is a multi-disciplinary open access archive for the deposit and dissemination of scientific research documents, whether they are published or not. The documents may come from teaching and research institutions in France or abroad, or from public or private research centers.
L'archive ouverte pluridisciplinaire HAL, est destinée au dépôt et à la diffusion de documents scientifiques de niveau recherche, publiés ou non, émanant des établissements d'enseignement et de recherche français ou étrangers, des laboratoires publics ou privés. 


\title{
Microchannel-connected SU-8 Honeycombs \\ by Single-step Projection Photolithography \\ for Positioning Cells on Silicon Oxide Nanopillar Arrays
}

\author{
Florian Larramendy ${ }^{1}$, Marie Charline Blatche ${ }^{2,3}$, Laurent Mazenq ${ }^{2,3}$, Adrian Laborde ${ }^{2,3}$, Pierre \\ Temple-Boyer ${ }^{2,3}$, Oliver Paul ${ }^{1}$ \\ ${ }^{1}$ Department of Microsystems Engineering (IMTEK), University of Freiburg, D-79115 Freiburg, \\ Germany \\ ${ }^{2}$ CNRS, LAAS, 7 avenue du colonel Roche, F-31400 Toulouse, France \\ ${ }^{3}$ University of Toulouse, UPS, LAAS, F-31400 Toulouse, France
}

\begin{abstract}
We report on the fabrication, functionalization and testing of SU-8 microstructures for cell culture and positioning over large areas. The microstructure consists of a honeycomb arrangement of cell containers interconnected by microchannels and centered on nanopillar arrays designed for promoting cell positioning. The containers have been dimensioned to trap single cells and, with a height of $50 \mu \mathrm{m}$, prevent cells from escaping. The structures are fabricated using a single ultraviolet photolithography exposure with focus depth in the lower part of the SU-8 resist. With optimized process parameters, microchannels of various aspect ratios are thus produced. The cell containers and microchannels serve for the organization of axonal growth between neurons. The roughly 2$\mu \mathrm{m}$-high and 500-nm-wide nanopillars are made of silicon oxide structured by deep reactive ion etching. In future work, beyond their cell positioning purpose, the nanopillars could be functionalized as sensors. The proof of concept of the novel microstructure for organized cell culture is given by the successful growth of interconnected PC12 cells. Promoted by the honeycomb geometry, a dense network of interconnections between the cells has formed and the intended intimate contact of cells with the nanopillar arrays was observed by scanning electron microscopy. This proves the potential of these new devices as tools for the controlled cell growth in an interconnected container system with well-defined three-dimensional geometry.
\end{abstract}


Keywords: Biosensor, SU-8 honeycomb structures, nanopillars, cell positioning, cell culture, interconnected neurons

\section{Introduction}

The analysis of neuronal cells arranged in networks of controlled geometry has grown rapidly over the past decade and stays important for biological studies and medical research. The positioning of individual cells has become a key technique for cell engineering applications such as cell therapy [1] and brain regeneration [2]. A further field of interest in this context is experimental neuroscience [3] where activity in neuronal networks is monitored by sensors responding to electrochemical potential changes near individual neurons. Extracellular recordings are often performed using single electrodes or multi-electrode arrays (MEA) [4], or using semiconductor devices such as transistors and capacitors on silicon chips [5, 6]. However, extracellular recordings reflect the size of the cleft [7] between the cells and electrodes, and thus suffer from reduced signal strength and quality. To circumvent this drawback, techniques for intracellular recording such as patch clamp recording have been developed [8]. This technique requires considerable expertise and has been applied dominantly to cells in suspension rather than to adherent cells or cellular networks. Inspired by previous work at Stanford University [9], we focus in this paper on nanopillars to promote the interaction with neuronal cells. The authors of Ref. 9 have indeed shown that nanopillars improve the neuron-to-electrode contact and offer the advantages of long-term measurements, high sensitivity, and minimal invasiveness [10].

In addition to implementing appropriate sensor, it is beneficial to incite cells to adhere and develop at intended locations. Several categories of methods have been explored with this purpose in mind. They include structured surface functionalization $[11,12,13]$, microfluidic guiding and trapping $[14,15,16]$, negative dielectrophoresis (nDEP) $[17,18]$, and micropatterned topographic constraints for cell growth [19, 20, 21].

Surface functionalization has been achieved for example by microcontact printing using polydimethylsiloxane (PDMS) stamps [13]. However in this well-established technique aligning the PDMS stamp precisely with subjacent structures is challenging. Furthermore the technique cannot be applied to high-topography surfaces.

Microfluidic structures [16] also make it possible to guide cells to intended locations. The photolithography-based fabrication of microfluidic components avoids the disadvantages of microcontact printing. Overall, nevertheless, the approach is more demanding since it requires potentially complex microfluidic system control. Similarly, nDEP requires an electrical system control. Furthermore electrical field gradients have be applied continuously or repeatedly to the cells to prevent them from migrating away from their locations. 
Finally the topography of microstructure has been taken advantage of to influence [22] or guide cell growth [23]. In this work, in order to position cells on nanopillars we choose to rely on such microstructures made of thick photoresist. Several techniques have in the past allowed to successfully fabricate three-dimensional (3D) photoresist structures. These include interference lithography [24] and dosage-controlled optical exposure [25] which both involve dedicated exposure equipment. An alternative is lamination [26] which necessitates multiple iterations of the same process sequence. Finally two-photon polymerization [27] has proven useful as well. This serial exposure technique is well-suited for small structures, but it is time-consuming for the preparation of large numbers of samples or large-area substrates.

In this work we therefore set out to develop a technique that enables polymer microstructures to be fabricated on large substrates with delicate high-topography surfaces, using simple equipment and in a single exposure step. The technique has been developed with a particular configuration in mind where honeycomb arrangements of polymer microcontainers for cells are centered on nanopillar arrays and interconnected by microchannels. These structures are produced using a single photolithography mask, a single photoresist layer and standard commercial equipment for optical projection lithography [28], as explained in detail in Section 2. As the photoresist, we use SU-8, a common epoxy-based negative photoresist [29]. The realization of nanopillars to simulate sensors is presented in Section 2 as well. In Section 3 experiments with PC12 cells [30], a cell line derived from a pheochromocytoma of the rat adrenal medulla, demonstrate the effectiveness of the honeycomb structures in position cells on nanopillars. The viability of cells is tested by a fluorescence "live-dead" test, and PC12 cell growth within the honeycomb structures and on nanopillars is observed by scanning electron microscopy (SEM).

\section{Materials and Methods}

\subsection{Stepper technology}

Optical lithography by projection, also called stepper lithography, consists of exposing a photosensitive resist with a thickness $t_{\mathrm{PR}}$ through a complex optical system acting as a reduction lens. The principle is illustrated in figure 1(a). The photolithographic reticle (the mask) is usually placed far from the substrate ( 0.2 to 1 meter) compared to conventional mask aligners where the mask-substrate distance is typically less than $200 \mu \mathrm{m}$. The mask pattern is illuminated by an appropriate light source and optically projected onto the photosensitive layer with a down-scaling factor up to 50:1. The projection optics transfers the plane of the mask with its light and dark 
patterns into the so-called focal plane, lying usually within the photoresists, where an image of the original pattern is obtained. In the language of geometrical optics, each point of the image is formed by the convergence of a projection cone with an opening angle $\theta_{0}$ in the space between the projection lens and the photoresist. The projection system used in this study is a Canon FPA 3000 i4 stepper from ASML company (Veldhoven, Netherlands). The selected down-scaling factor was 5:1, while in Fig. 1(a), for better clarity it was chosen to be only $2: 1$. The UV wavelength is $365 \mathrm{~nm}$. The value of $\theta_{0}$ is $39.05^{\circ}$.

The main parameters of the stepper lithography process are the exposure dose $D_{\text {exp }}$ and the focus depth $d_{\mathrm{f}}$. Steppers present chip-to-chip variations of $D_{\text {exp }}$ by typically $\pm 3 \%$. In our experiments, controlled exposure dose variations are obtained by combining the exposure duration and the illumination aperture using a constant UV light intensity defined by the lamp power.

On the other hand, the focus depth $d_{\mathrm{f}}$ can be modified by changing the position of the wafer with respect to the optical lens, i.e. by changing the lens-wafer distance by the shift $\Delta z$ with respect to a reference distance set automatically by the stepper. This is illustrated in figure 1 . In the left half of figure 1(a) and in figure 1(b), the reticle is focused in the middle of the photoresist, defining a focus depth $d_{\mathrm{f}, \mathrm{m}}$, corresponding to a lens-wafer distance shift $\Delta z_{\mathrm{m}}$. The dark line segment in the focal plane in figure 1(a) and the horizontal two-sided arrow in figure 1(b) indicate the extension of the image of the original dark mask pattern.

As indicated by the illumination cone in figure 1(c), due to refraction within the photoresists the conical illumination distribution is characterized by the angle $\theta_{1}=22.19^{\circ}$. This value is related to $\theta_{0}$ by $n \sin \theta_{1}=\sin \theta_{0}$, where $n$ denotes the refractive index of the photoresist film. For SU-8, we have $n=1.668$ at $365 \mathrm{~nm}$ [31]. As a result of the refractive property of the photoresist, upon a lens-wafer distance variation by $\Delta \mathrm{z}, d_{\mathrm{f}}$ is varied by $\left(\tan \theta_{0} / \tan \theta_{1}\right) \times \Delta z \approx 2 \Delta z$. If $d_{\mathrm{f}}$ and $\Delta z$ are below $d_{\mathrm{f}, \mathrm{m}}$ and $\Delta z_{\mathrm{m}}$, respectively, the focal plane lies in the lower half of the photoresist, as illustrated in figure 1(c).

Based on the available information, it was unfortunately not possible to unambiguously identify the focus location $d_{\mathrm{f}}=0$ corresponding to some reference lens-wafer distance with $\Delta z=0$, which in addition may depend on the used photoresist. The inspection of a larger number of structures observed as a function of $d_{\mathrm{f}}$ in the case of $50-\mu \mathrm{m}$-thick SU-8 leads us to the conclusion that for this resist, $\Delta z=d_{\mathrm{f}}=0$ corresponds to a focal plane near the upper resist surface. As a consequence, in the following all focus depth values for focal planes within the photoresist are negative numbers; the more negative they are, the deeper the focal plane lies below the photoresist surface. The corresponding $\Delta z$ values are also negative which tells that the wafer was shifted towards the projection lens. 


\subsection{Focus depth variation}

By setting $d_{\mathrm{f}}<d_{\mathrm{f}, \mathrm{m}}$ by decreasing the focus depth towards more negative values as shown in figure 1(c), i.e. towards and even below the substrate surface, it was possible to focus the reticle in the lower part of the resist film, leading to a UV exposure imbalance between its upper and lower parts. Different shades in figures 1(b) to (e) represent different exposure doses $D_{\text {exp. }}$ Darkest shades correspond to the lowest dose, while areas of lightest shading experience the strongest exposures. Which volumes of photoresist are removed by the photoresist development depends on whether $D_{\text {exp }}$ has remained inferior (in the case of a negative photoresist) or is superior (for a positive

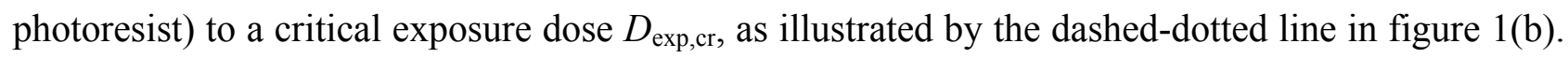
For the case of a negative photoresist such as SU-8 used in this study, the resulting channel structure is shown figure 1(d). In contrast the illumination density in figure 1(c), shifted downward in comparison to figure 1(b), leads to a development result with a buried microchannel, as shown in figure 1(e).

Standard 6-inch p-doped $<100>$ silicon (Si) wafers were used as substrates for all the honeycomb microstructures fabricated in this study. A wet thermal oxidation to a silicon oxide $\left(\mathrm{SiO}_{2}\right)$ thickness of $2.2 \mu \mathrm{m}$ is carried out first in a thermal furnace (Centrotherm, Blaubeuren, Germany) for 8 hours at $1150^{\circ} \mathrm{C}$ at atmospheric pressure with an $\mathrm{H}_{2}: \mathrm{O}_{2}$ ratio of $8: 1$. Then a 20 -nm-thick silicon nitride $\left(\mathrm{Si}_{3} \mathrm{~N}_{4}\right)$ is deposited by plasma-enhanced chemical vapor deposition (PECVD) in a PECVD STS Multiplex tool (STS, Bristol, UK) using HF plasma (frequency $13,56 \mathrm{MHz}$, power $15 \mathrm{~W}$ ) at $300^{\circ} \mathrm{C}$ for $105 \mathrm{~s}$ at 1 Torr pressure with the following gas fluxes: $\mathrm{SiH}_{4}(30 \mathrm{sccm}), \mathrm{NH}_{3}(40 \mathrm{sccm}), \mathrm{N}_{2}(1200$ sccm). This pre-processing serves to implement the nanopillar process, presented in Section 2.3. This entire study of interconnected honeycomb structures is realized with SU-8 with a thickness fixed at $t_{\mathrm{PR}}=50 \mu \mathrm{m}$. The stepper allows to set $d_{\mathrm{f}}$ and $D_{\mathrm{exp}}$ individually for each chip on the wafer. In this study, $d_{\mathrm{f}}$ was varied from chip to chip in the wafer $x$ direction by programming $\Delta z$ to run from $-50 \mu \mathrm{m}$ to $-7.5 \mu \mathrm{m}$ in steps of $2.5 \mu \mathrm{m}$. On the other hand, $D_{\text {exp }}$ was varied in the $y$ direction from $900 \mathrm{~J} / \mathrm{m}^{2}$ to $1250 \mathrm{~J} / \mathrm{m}^{2}$ in steps of $50 \mathrm{~J} / \mathrm{m}^{2}$. Two distinct honeycomb designs (Designs 1 and 2) shown in figures 2(a) and (b), respectively, were explored. Black and white areas correspond to the opaque and transparent mask areas, respectively. In the two designs, hexagonal cell containers are pairwise connected by interconnections giving rise, when suitably exposed, to buried microchannels. The designed length $l$ and width $w$ of the interconnection structures and diameter $r$ of the cell containers are indicated in figure 2. In the case of Design 1, by the 5:1 reduction these dimensions result in dimensions in the focal plane of $l=30 \mu \mathrm{m}, w=10 \mu \mathrm{m}$ and $r=40 \mu \mathrm{m}$. In contrast, Design 2 has shorter interconnections with $l=10 \mu \mathrm{m}$ and $w=10 \mu \mathrm{m}$, but a larger cell 
container diameter $r=60 \mu \mathrm{m}$. Results for these two designs are presented in Section 3.1. Each design was realized on three wafers.

The post exposure bake (PEB) is performed on a hot plate with a controlled temperature history. The PEB starts with a rising ramp of $10^{\circ} \mathrm{C} / \mathrm{min}$ up to $95^{\circ} \mathrm{C}$, proceeds with a hold period of $3 \mathrm{~min}$. at this temperatures, and is finished with descending ramp of $-5^{\circ} \mathrm{C} / \mathrm{min}$ down to room temperature. This process has been previously investigated at the authors' lab for $50-\mu \mathrm{m}$-thick SU-8. Since these previous studies indicated that the PEB parameters have little effect on the final SU-8 structures, we decided to leave the PEB parameters unchanged at the above values throughout the present study. The focus here is therefore only on the effect of $d_{\mathrm{f}}$ and $D_{\text {exp }}$. Finally, the exposed and post-baked SU-8 layer is developed in standard SU-8 Developer for $20 \mathrm{~min}$.

\subsection{Nanopillar realization}

Prior to SU-8 processing, nanopillar arrays were fabrication using the $2.2-\mu \mathrm{m}$-thick thermal $\mathrm{SiO}_{2}$ on the wafers. A primer and positive photoresist ECI from Microchem, Newton, USA of $2.6 \mu \mathrm{m}$ thickness were successively spun onto the wafer. Next, the photoresist was pre-annealed, exposed, post-annealed and developed as a protection against the subsequent etching. It was performed by reactive ion etching (RIE) using the Electrotech Omega201 etcher from Trikkon, Canada. RIE relied on trifluoromethane $\left(\mathrm{CHF}_{3}\right)$ at $5 \mathrm{mTorr}$ for $45 \mathrm{~s}$ to etch the primer, followed by 14 min to etch $2 \mu \mathrm{m}$ of $\mathrm{SiO}_{2} . \mathrm{O}_{2}$ plasma and Piranha etches were used to remove the photoresist without damaging the nanopillars. Nanopillars are typically $300 \mathrm{~nm}$ and $700 \mathrm{~nm}$ in diameter at the top and base of the pillars, respectively, and $2 \mu \mathrm{m}$ in height, as shown in figure 3(a). The Omega201 etcher anisotropically etches $\mathrm{SiO}_{2}$ and thus caused the slope of the nanopillars to be about $5.7^{\circ}$. Three types of nanopillar arrays are tested, as indicated in figure $3(\mathrm{~b})$ by the areas denoted A, B and C with hexagonal arrangements of 7, 37, and 91 nanopillars, respectively, with a pitch of $1 \mu \mathrm{m}$. To complete the nanopillar fabrication, a $\mathrm{Si}_{3} \mathrm{~N}_{4}$ layer of $20 \mathrm{~nm}$ thickness is deposited by PECVD. $\mathrm{Si}_{3} \mathrm{~N}_{4}$ has been found to be non-toxic to rat brain slices [32] and to allow the inflammation-free proliferation of a human osteoblast cell line [33].

\subsection{PC12 cell cultures}

The experiments were performed using cells that had undergone fewer than five passages. As described previously [34, 35], PC12 cells (American Type Culture Collection CRL-1721, from the Duke Comprehensive Cancer Center, Durham, NC) were seeded onto poly-D-lysine and laminincoated chips with Dubelcco's Modified Eagle Medium (DMEM) (HyClone, USA) supplemented 
with 10\% horse serum (HyClone), 5\% fetal bovine serum (HyClone), and $50 \mu \mathrm{g} / \mathrm{mL}$ penicillin streptomycin (Invitrogen, Carlsbad, CA). 20,000 cells $/ \mathrm{cm}^{2}$ were deposited onto the chips. Incubation was carried out under standard conditions for $\mathrm{PC} 12$ cells, i.e., $5 \% \mathrm{CO}_{2}$ at $37^{\circ} \mathrm{C}$. For five consecutive days, the medium was replaced daily by fresh medium of the same composition except for the substitution of the horse serum by $150 \mathrm{ng} / \mathrm{mL}$ of nerve growth factor (NGF) (HyClone). This duration is required for the development of cell extensions. In this study, we did not distinguish between axons and dendrites and subsequently term all extensions neurites.

\subsection{Cell viability assessment}

Neuronal cells were visualized with the "live-dead" test no. L-3224 from Life Technologies where viable cells are labeled with calcein-AM detectable as green fluorescence, whereas dead cells are labeled with ethidium iodide producing a red fluorescence. In more detail, the cell viability assessment was performed as follows. After three days in culture, chips carrying 3D SU-8 microstructures were incubated in a mixture of calcein-AM and ethidium homodimer-1 diluted in a phosphate buffered saline (PBS) medium with $\mathrm{pH}=7.4$ for one hour in a humidified chamber at $37^{\circ} \mathrm{C}$ with $5 \% \mathrm{CO}_{2}$. The silicon chips were mounted with FluorSave reagent (Calbiochem, San Diego, CA, USA) and viewed with the upright microscope BX51TF (Olympus, Tokyo, Japan) with a fluorescent source (Lumencor, Beaverton, USA) and an iXon+ camera (Andor, Belfast, UK). Excitation for live cells is at $494 \mathrm{~nm}$ and cells emit at $517 \mathrm{~nm}$ in green fluorescence. For dead cells, excitation is at $517 \mathrm{~nm}$ and cells emit at $617 \mathrm{~nm}$ in red fluorescence.

\subsection{Neuronal cell fixation for electron microscopy observation}

After checking the viability of cells on each chip using epifluorescence microscopy, the culture medium was removed. For fixation, the chips were rinsed with PBS solution and then incubated in $4 \%$ glutaraldehyde solution (Sigma-Aldrich) for 12 hours at $4{ }^{\circ} \mathrm{C}$. Then, the glutaraldehyde solution was removed and the chips were rinsed with PBS solution. For cell dehydration, we used a cascade of aqueous ethanol solutions with increasing ethanol concentrations. The cells were therefore incubated in 50\%, 70\%, 90\% and 100\% ethanol for 10 minutes each. After the last dehydration step, the fixed samples were air-dried at room temperature for a day to remove alcohol residues before inspection using a scanning electron microscope (SEM). Amazingly good-looking cells are the result, without apparent degradation or collapse of their delicate structure as might be expected from a drying procedure without critical-point drying. This preparation method for SEM observation has already been successful applied in studies of nanoscale topography on neurite 
development in PC12 cells [36] and of silicon nitride windows for electron microscopy of whole cells [37]. Further technical details have been reported in Ref. 38.

\section{Results and discussion}

\subsection{Honeycomb cell container characterization}

First, the variation of $d_{\mathrm{f}}$ allowed us to define microchannels of various geometries. Roughly speaking, for a fixed exposure dose, when the focus depth is too negative $\left(d_{\mathrm{f}}<-80 \mu \mathrm{m}\right)$, it was found that the microchannel is blocked, as shown in figure $4(\mathrm{a})$, while high values $\left(d_{\mathrm{f}}>-20 \mu \mathrm{m}\right)$ lead to microchannels open at the surface (figure 4(c)). For $d_{\mathrm{f}}$ values around $-50 \mu \mathrm{m}$, buried microchannels are obtained (figure 4(b)). Depending on the exposure parameters, they can attain various shapes. Similarly for decreasing exposure dose $D_{\exp }$ at fixed focus depth, we observe a transition from blocked channels via buried channels to surface channels. For a more detailed study of microchannel configurations, three geometric parameters were measured: microchannel width and height, and cover length achievable with Designs 1 and 2 with $50-\mu \mathrm{m}$-thick SU-8 layers as a

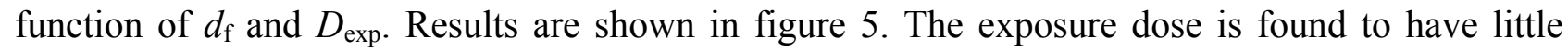
influence on the width and the height of the microchannels, but has a significant impact on the cover length, which increases with $D_{\text {exp }}$. On the other hand, $d_{\mathrm{f}}$ is found to be the key parameter determining the microchannel cross-section. Indeed, $d_{\mathrm{f}}$ directly allows to control the microchannel height; this parameter increases with decreasing value of $d_{\mathrm{f}}$ from buried to surface channels. The microchannel width is influenced as well by $d_{\mathrm{f}}$ and presents a maximum value around $-50 \mu \mathrm{m}$ depending on the design. A maximum is also observed for the microchannel cover length at $d_{\mathrm{f}}=-75 \mu \mathrm{m}$ for Design 2. The design of the microchannel also has an impact on the parameter choices. Design 1 has a length-to-width ratio of 3 on the reticle while Design 2 has a ratio of 1 and as a consequence is more sensitive to $d_{\mathrm{f}}$ than Design 1 . These results show that there are not only

one configuration to create microchannels but also different combinations of $d_{\mathrm{f}}$ and $D_{\text {exp. As a }}$ consequence, various design and the microchannel shape can be realized.

As a result, by tuning both parameters, it is possible to control the three-dimensional geometry of the microchannels. After optimizing $D_{\exp }$ and $d_{\mathrm{f}}$, networks of honeycomb structures with microchannels were successfully fabricated at wafer scale into $50-\mu \mathrm{m}$-thick SU-8 layer. We choose to fix $d_{\mathrm{f}}$ to $-60 \mu \mathrm{m}$ and $D_{\exp }$ to $1000 \mathrm{~J} / \mathrm{m}^{2}$ for the subsequent experiments.

In addition, the realization of honeycomb cell container structures was tested on three different substrates, namely monocrystalline silicon as well as $\mathrm{SiO}_{2}$ and $\mathrm{Si}_{3} \mathrm{~N}_{4}$ covered wafers in order to assess the influence of the substrate reflection. This study showed that the substrate reflection has a 
minor impact on the shape of the microchannel. However in the case of silicon substrate, the stronger reflection caused a dark halo to locally appear at the base of the SU-8 layer, as evidenced by figure 4 . This halo was successfully removed by increasing the SU-8 development time from 10 to 30 minutes.

\subsection{Cells on nanopillars without SU-8 cell containers}

As described in Section 2.4, PC12 cell were cultured on chips with nanopillars without SU-8 cell containers for 5 days. The live-dead test revealed that over $90 \%$ of the cells were alive. It was not possible to clearly observe cells on nanopillars using fluorescence and optical microscopies; for this reason we used the SEM instead. To this end, cells were fixed using the technique introduced in Section 2.5. In the SEM the chips were tilted at $30^{\circ}$ in order to allow the shapes of the nanopillars and cells to be observed simultaneously. Different chips with the three types of nanopillar arrangements $\mathrm{A}$ to $\mathrm{C}$ gave rise to the following conclusions.

First, it was determined that nanopillars do not fundamentally affect the cell culture growth. This conclusion was drawn on the basis of the fact that similar cell densities were observed on areas without nanopillars and with the different types of nanopillar arrays.

Second, the size of the nanopillar arrays was found to have an impact on the number of cell-tonanopillar connections. By cell-to-nanopillar connections we mean that a neurite of a cell passes over a nanopillar array and is visibly attached to at least one nanopillar or that a cell is in direct contact with a nanopillar array or resides even on top of it. The smallest array, A, promotes the lowest number of such connection. Nevertheless neurites exhibit a tendency to attach to nanopillars when they are close to a nanopillar array. Neurites connected to nanopillars in arrays A, B, and C represent $16 \pm 2 \%, 38 \pm 3 \%$, and $42 \pm 3 \%$, respectively, of all neurites. Understandably, larger arrays offer a higher probability of intercepting neurites. Conversely neurites do not seem to be particularly repelled by their interaction with the arrays.

Some cells grew with their cell body in direct contact with nanopillar arrays and some are even placed on top of nanopillar arrays (figure 6(a)). Nevertheless these cases represent less than $10 \%$ of the cells connected to nanopillars. The remaining fraction of cells preferred to grow on the planar substrate beside the nanopillar arrays and to limit their interaction with the arrays to their neurites.

\subsection{Cell prepositioning on nanopillars by interconnected cell containers}

To promote the cell growth on top of the nanopillar arrays, honeycomb cell container structures were thus realized around nanopillar arrays, as shown in figure 6. Design 1 and 2 cell container 
structures were realized around all three sizes of nanopillar arrays. To overcome the potential cytotoxicity of SU-8 [39], we followed a four-step protocol to turn the SU-8 into a biocompatible material. The first step is the chemical treatment with SU-8 developer in order to remove all resist residues across microchannels by SU-8 over-development. The second step consisting of a 1-min UV exposure at $365 \mathrm{~nm}$ wavelength has the purpose of reinforcing the polymer chain links within the SU-8 photoresist across its entire thickness. The third step is a thermal anneal at $150^{\circ} \mathrm{C}$ for three days to eliminate all solvents trapped within the SU-8. The final step is an $\mathrm{O}_{2}$ plasma treatment to render the SU-8 surface hydrophilic [40]. Quantitative SEM observations before and after the specific treatment showed that this protocol does not significantly affect the final dimensions of SU-8 microstructures nor the adhesion between the SU-8 layer and the substrate, nor does it introduce any cracks in the SU-8 layer.

After performing the protocol, we deposited poly-D-lysine and coated laminin in order to create a substrate favorable to cell expansion and neuronal differentiation [41]. After cell culture, chips were first observed by optical microscopy to map the location of cells in containers. This allowed to assess possible cell losses during the subsequent steps. Second, the live-dead test was realized on cells in the SU-8 microstructures to observe whether cells had survived in the containers. However this technique proved to offer limited value due to the concurrent fluorescence of SU-8. Thirdly therefore, to validate the viability of cells in this case, we defined two parameters observable both in the SEM: cells needed to be attached on the substrate and to show no obvious sign of degradation. A flattened cell shape as well as cracks and other obvious damage were taken as such signs.

From the observations we conclude that PC12 cells are successfully prepositioned by the cell container honeycomb and develop neurites across the microchannels. Neurites pass over nanopillars or finish their trajectory on nanopillars if they do not find another neurite or cell to connect to. Figure 6 illustrates neuron and neurite growth on nanopillars and across microchannels. The number of cells per $\mathrm{cm}^{2}$ is decreased in comparison with chips without cell containers because a large fraction of the overall area is occupied by SU-8; in the case of Design 1 the decrease is about $50 \%$; it is about 33\% for Design 2. Despite this absolute overall decrease in cell population, the fraction of cells connected to nanopillars directly or through neurites is significantly increased. These results are quantitatively summarized in figure 7 showing the percentages of cells connected to nanopillars of each type, surrounded or not by SU-8 cell containers of both Designs 1 and 2. As a conclusion, in every case the SU-8 cell containers significantly increase the fraction of cells connected to nanopillars. 


\section{Conclusion}

In this paper, UV stepper projection lithography was used in a non-standard way to fabricate SU-8 based cell containers arranged in a honeycomb structure and connected by microchannels. The lithography required a single exposure. Advantages of the single-step SU-8 projection lithography approach is its simplicity, leading to a saving of time (one exposure) and material (one mask), and the considerable freedom of design it offers in view of the two main exposure parameters, $d_{\mathrm{f}}$ and $D_{\text {exp }}$, studied in this work. By investigating their influence on two different designs, the technique was optimized to produce cell containers connected by buried channels. The study of cells cultured on nanopillar arrays of three different sizes combined with the two honeycomb designs showed that the connected cell containers increased the number of connections between cells and nanopillars in comparison with nanopillar arrays on bare substrates, despite a reduction in the absolute cultured cell density. This study is a preparatory step towards intracellular recordings from neuronal networks of hexagonal or other geometry. The structures may support our understanding of neurocomputational processes and the realization of labs-on-a-chip for medical analysis.

\section{Acknowledgments}

Technical support by A. Lecestre at LAAS-CNRS (France) and by Prof. S. Takeuchi (IIS, the University of Tokyo, Japan) and his team and financial support by project EUJO-LIMMS (no. 295089) funded by the EU 7th Framework Program are gratefully acknowledged.

\section{References}

[1] Yang K, Lee J S, Kim J, Lee Y B, Shin H, Um S H, Kim J B, Park K I, Lee H, and Cho S-W, 2012 Polydopamine-mediated surface modification of scaffold materials for human neural stem cell engineering Biomaterials, 33, 6952-6964

[2] Forraz N, Wright K E, Jurga M, and McGuckin C P, 2013 Experimental therapies for repair of the central nervous system: stem cells and tissue engineering Journal of Tissue Engineering and Regenerative Medicine, 7, 7, 523-536.

[3] Ballini M, Müller J, Livi P, Chen Y, Frey U, Stettler A, Shadmani A, Viswam V, Jones I L, Jäckel D, Radivojevic M, Lewandowska M K, Gong W, Fiscella M, Bakkum D J, Heer F, and Hierlemann A, 2014 A 1024-Channel CMOS Microelectrode Array With 26,400 Electrodes for Recording and Stimulation of Electrogenic Cells In Vitro IEEE J. Solid-St Circ., 49, 11, 2705-2719. 
[4] Hogberg H T, Sobanski T, Novellino A, Whelan M, Weiss D G, and Bal-Price A K, 2011 Application of micro-electrode arrays (MEAs) as an emerging technology for developmental neurotoxicity: Evaluation of domoic acid-induced effects in primary cultures of rat cortical neurons NeuroToxicology, 32, 158-168.

[5] Erikson J, Tooker A, Tai Y-C, and Pine J, 2008 Caged neuron MEA: A system for long-term investigation of cultured neural network connectivity J. Neurosci. Meth., 175, 1-16.

[6] Hierlemann A, Frey U, Hafizovic S, and Heer F, 2011 Growing Cells Atop Microelectronic Chips: Interfacing Electrogenic Cells In Vitro With CMOS-Based Microelectrode Arrays P. IEEE 99, 252-284.

[7] Voelker M, and Fromherz P, 2005 Signal Transmission from Individual Mammalian Nerve Cell to Field-Effect Transistor Small, 1, 2, $206-210$.

[8] Edwards F A, Konnerth A, Sakmann B, and Takahashi T, 1989 A thin slice preparation for patch clamp recordings from neurones of the mammalian central nervous system Eur. J. Phycol., 414, 600-612.

[9] Xie C, Hanson L, Xie W, Lin Z, Cui B, and Cui Y, 2010 Noninvasive Neuron Pinning with Nanopillar Arrays Nano Lett., 10, 4020-4024.

[10] Xie C, Lin Z, Hanson L, Cui Y, and Cui B, 2012 Intracellular recording of action potentials by nanopillar electroporation Nat. Nanotechnol., 7, 185-190.

[11] Heller D A, Gargab V, Kelleher K J, Lee T-C, Mahbubani S, Sigworth L A, Lee T R, and Rea M A, 2005 Patterned networks of mouse hippocampal neurons on peptide-coated gold surfaces Biomaterials, 26, 883-889.

[12] Offenhäusser A, Böcker-Meffert S, Decker T, Helpenstein R, Gasteier P, Groll J, Möller M, Reska A, Schäfer S, Schulte P, and Vogt-Eisele A, 2007 Microcontact printing of proteins for neuronal cell guidance Soft Matter, 3, 290-298.

[13] Qin D, Xia Y, and Whitesides G M, 2010 Soft lithography for micro- and nanoscale patterning Nat. Protoc., 5, 3, 491-502.

[14] Rhee S W, Taylor A M, Tu C H, Cribbs D H, Cotman C W, and Jeon N L, 2005 Patterned cell culture inside microfluidic devices Lab Chip, 5, 102-107.

[15] Lin L, Chu Y-S, Thiery J P, Lim C T, and Rodriguez I, 2013 Microfluidic cell trap array for controlled positioning of single cells on adhesive micropatterns Lab Chip, 13, 714-721.

[16] Dertinger S K W, Jiang X, Li Z, Murthy V N, Whitesides G M, 2002 Gradients of substratebound laminin orient axonal specification of neurons Gradients of substrate-bound laminin orient axonal specification of neurons P.Natl. Acad. Sci. USA, 99, 12542-12547.

[17] Frénéa M, Faure S P, Le Pioufle B, Coquet P, and Fujita H, 2003 Positioning living cells on a high-density electrode array by negative dielectrophoresis Mat. Sci. Eng. C, 23, 5, 597-603. 
[18] Yasukawa T, Yamada J, Shiku H, Mizutani F, and Matsue T, 2013 Positioning of cells flowing in a fluidic channel by negative dielectrophoresis Sensor. Actuat. B-Chem., 186, 9-16.

[19] Marelli M, Gadhari N, Boero G, Chiquet M, and Brugger J, 2014 Cell force measurements in 3D microfabricated environments based on compliant cantilevers Lab Chip, 14, 286-293.

[20] Rosenthal A, Macdonald A, and Voldman J, 2007 Cell patterning chip for controlling the stem cell microenvironment Biomaterials, 28, 21, 3208-3216.

[21] Choi J H, Lee H, Jin H K, Bae J-S and Kim G-M, 2012 Micropatterning of neural stem cells and Purkinje neurons using a polydimethylsiloxane (PDMS) stencil Lab Chip, 12, 5045-5050.

[22] Mahoney M J, Chen R R, Tan J, and Saltzman W M, 2005 The influence of microchannels on neurite growth and architecture Biomaterials, 26 (7), 771-778

[23] Béduer A, Gonzales-Calvo I, Vieu C, Loubinoux I, and Vaysse L, 2013 Investigation of the Competition Between Cell/Surface and Cell/Cell Interactions During Neuronal Cell Culture on a Micro-Engineered Surface Macromol. Biosci., 13, 11, 1546-1555.

[24] Campbell M, Sharp D, Harrison M, Denning R, and Turberfield A, 2000 Fabrication of photonic crystals for the visible spectrum by holographic lithography Nature, 404, 53-56.

[25] Hirai Y, Sugano K, Tsuchiya T, and Tabata O, 2010 Embedded Microstructure Fabrication Using Developer-Permeability of Semi-Cross-Linked Negative Resist J.Microelectromech. S., 19, 1058-1069.

[26] Abgrall P, Lattes C, Conedera V, Dollat X, Colin S, and Gue A M, 2005 A novel fabrication method of flexible and monolithic 3D microfluidic structures using lamination of SU-8 films $J$. Micromech. Microeng., 16, 113-121.

[27] Greiner A M, Richter B, and Bastmeyer M, 2012 Micro-Engineered 3D Scaffolds for Cell Culture Studies Macromol. Biosci., 12, 1301-1314.

[28] Larramendy F, Mazenq L, Temple-Boyer P, and Nicu L, 2012 Three-dimensional closed microfluidic channel fabrication by stepper projection single step lithography: the diabolo effect Lab Chip, 12, 387-390.

[29] Zhang J, Tan K L, and Gong H Q, 2001. Characterization of the polymerization of SU-8 photoresist and its applications in micro-electro-mechanical systems (MEMS) Polym. Test., 20, 693-701.

[30] Hsiao Y-S, Lin C-C, Hsieh H-J, Tsai S-M, Kuo C-W, Chu C-W, and Chen P, 2011 Manipulating location, polarity, and outgrowth length of neuron-like pheochromocytoma (PC-12) cells on patterned organic electrode arrays Lab Chip, 11, 3674-3680.

[31] Del Campo A, and Greiner C, 2007 SU-8: a photoresist for high-aspect-ratio and 3D submicron lithography J. Micromech. Microeng.17, 81-95. 
[32] Kristensen B W, Noraberg J, Thiebaud P, Koudelka-Hep M, and Zimmer J, 2001 Biocompatibility of silicon-based arrays of electrodes coupled to organotypic hippocampal brain slice cultures Brain Res., 896, 1-17.

[33] Sohrabi A, Holland C, Kue R, Nagle D, Hungerford D S, and Frondoza C G, 2000 Proinflammatory cytokine expression of IL-1b and TNF-a by human osteoblast-like MG-63 cells upon exposure to silicon nitride in vitro J. Biomed. Mater. Res., 50, 43-49.

[34] Qiao D, Seidler F J, Violin J D, and Slotkin T A, 2003 Nicotine is a developmental neurotoxicant and neuroprotectant: stage-selective inhibition of DNA synthesis coincident with shielding from effects of chlorpyrifos Dev. Brain Res., 147, 183-190.

[35] Song X, Violin J D, Seidler F J, and Slotkin T A, 1998 Modeling the Developmental Neurotoxicity of Chlorpyrifosin Vitro: Macromolecule Synthesis in PC12 Cells Toxicol. Appl. Pharm., 151, 182-191.

[36] Haq F, Anandan V, Keith C, and Zhang G, 2007 Neurite development in PC12 cells cultured on nanopillars and nanopores with sizes comparable with filopodia Int. J. Nanomedicine, 2(1), $107-$ 115.

[37] Ring E A, Peckys D B, Dukes M J, Baudoin J P, and De Jonge N, 2011 Silicon nitride windows for electron microscopy of whole cells J. Microsc., 243, 273-283.

[38] Bozzola J J, and Russell L D, 1999 Electron Microscopy Principles and Techniques for Biologists, Jones and Bartlett Publishers, Boston.

[39] Vernekar V N, Cullen D K, Fogleman N, Choi Y, Garcia A J, Allen M G, Brewer G J, and LaPlaca M C, 2008 SU-8 2000 rendered cytocompatible for neuronal bioMEMS applications $J$. Biomed. Mater. Res-A 89, 138-151.

[40] Walther F, Davydovskaya P, Zürcher S, Kaiser M, Herberg H, Gigler A M, and Stark R W, 2007 Stability of the hydrophilic behavior of oxygen plasma activated SU-8 J. Micromech. Microeng. 17, 524-531.

[41] Qiana L, and Saltzman W M, 2004 Improving the expansion and neuronal differentiation of mesenchymal stem cells through culture Biomaterials 25, 1331-1337. 


\section{FIGURE CAPTIONS}

Figure 1. Principle of projection lithography and focus depth $d_{\mathrm{f}}$ variation for producing different types of microstructures in negative photoresist. (a) In projection lithography, an illuminated mask is projected onto a focal plane in the photoresist through a projection lens; the projection occurs with a cone of opening angle $\theta_{0}$ outside the photoresist; the photoresist-covered substrate can be shifted towards or away from the projection lens by a distance $\Delta z$, where $\Delta z_{\mathrm{m}}$ corresponds to a focal plane in the middle of the photoresist; $(\mathrm{b}, \mathrm{d})$ focal plane is at focus depth $d_{\mathrm{f}}=d_{\mathrm{f}, \mathrm{m}}$ in the middle of the photoresist of thickness $t_{\mathrm{PR}}$, (c,e) focus depth is shifted downward to value $d_{\mathrm{f}}<d_{\mathrm{f}, \mathrm{m}}$. Darker and lighter shades in photoresist in (b) to (d) indicate lower and higher effective exposure doses $D_{\exp }$, respectively; horizontal two-sided arrows in (b) and (c) indicate the sharp image of a dark structure of the projection reticle, projected within the photoresist with cones of opening angle $\theta_{1}$; (d) open and (e) buried microchannels obtained by the development of the photoresist in (b) and (c), respectively, after dissolution of photoresist of subcritical exposure $D_{\exp }<D_{\text {exp,cr. }}$.

Figure 2. Schematic of the two designs of interconnected honeycomb structures on the projection reticle. Dark and white areas indicate opaque and transparent areas on the reticle, respectively. Geometrical dimensions of (a) Design 1: $l=150 \mu \mathrm{m}, w=50 \mu \mathrm{m}, r=200 \mu \mathrm{m}$; of (b) Design 2: $l=50 \mu \mathrm{m}, w=50 \mu \mathrm{m}, r=300 \mu \mathrm{m}$. All dimensions are shrunk by a factor of 5 by projection.

Figure 3. Scanning electron micrograph of (a) a nanopillar and (b) a hexagonal array of 91 nanopillars termed type C. Smaller arrays with 37 (type B) and 7 (type A) nanopillars, as outlined in (b), were realized as well.

Figure 4. Scanning electron micrographs of cell container honeycombs of Design 2 obtained with different $d_{\mathrm{f}}$ values: containers (a) separated by thin walls, and connected by (b) buried and (c) surface microchannels.

Figure 5. Measured height (a,d), width (b,e) and length (c,f) of microchannels as a function of (a,b,c) exposure dose $D_{\exp }$ for $d_{\mathrm{f}}=-50 \mu \mathrm{m}$ and (d,e,f) as a function of focus depth $d_{\mathrm{f}}$ for $D_{\text {exp }}=1100 \mathrm{~J} / \mathrm{m}^{2}$ for Designs 1 (light grey symbols) and 2 (dark grey symbols) with $t_{\mathrm{PR}}=50 \mu \mathrm{m}$; plotted values represent averages and standard deviations from three wafers; lines are guides to the eye. 
Figure 6. Scanning electron micrographs of (a) a neuron on a nanopillar array with neurite growth, (b) a cell attached with several neurites to a nanopillar array, and (c,d) neurons pre-positioned on nanopillar arrays by a Design 2 honeycomb arrangement of cell containers, with neurites extending across microchannels. All nanopillar arrays in (a) to (d) are of type B.

Figure 7. Percentages of cells connected to nanopillar arrays of types A, B, and C on silicon substrates without SU-8 structures (darker grey bars) and embedded in SU-8 interconnected honeycomb structures of Designs 1 and 2 (lighter grey bar). Values represent averages and standard deviations from 48 chips. 


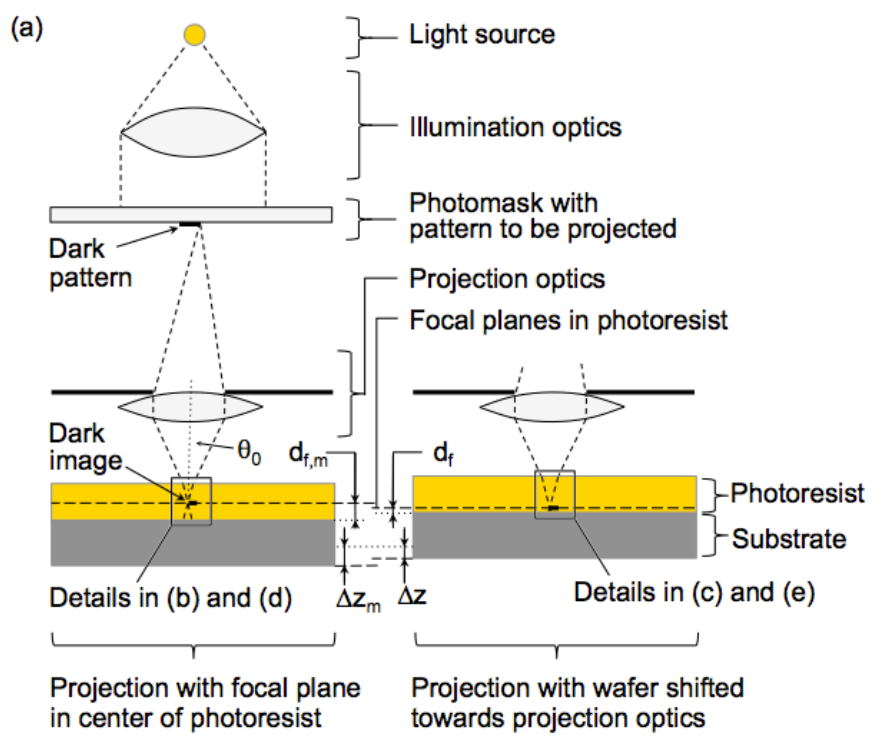

(b)

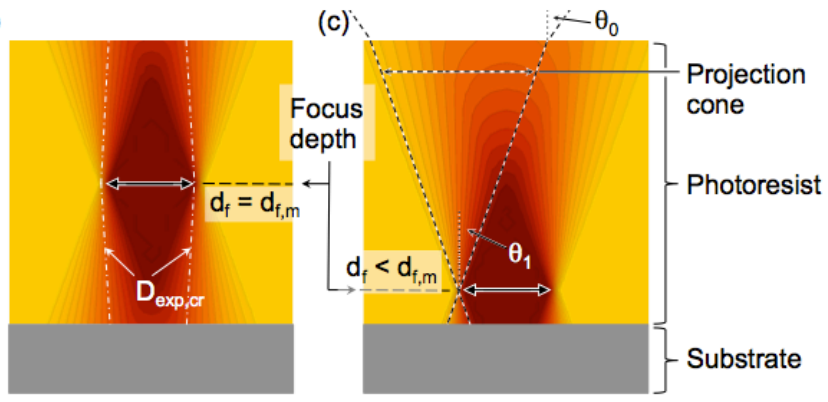

(d)

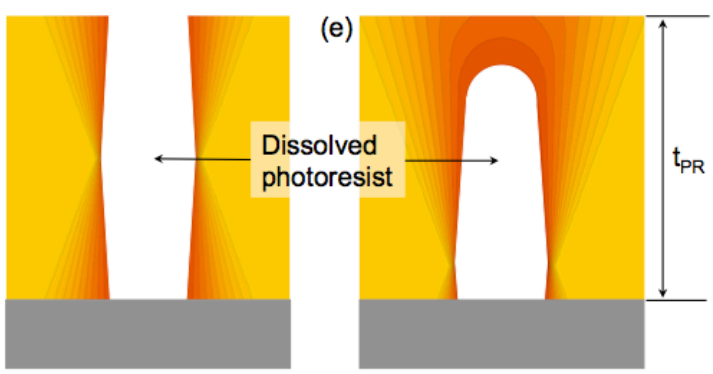

Figure 1. Principle of projection lithography and focus depth $d_{\mathrm{f}}$ variation for producing different types of microstructures in negative photoresist. (a) In projection lithography, an illuminated mask is projected onto a focal plane in the photoresist through a projection lens; the projection occurs with a cone of opening angle $\theta_{0}$ outside the photoresist; the photoresist-covered substrate can be shifted towards or away from the projection lens by a distance $\Delta z$, where $\Delta z_{\mathrm{m}}$ corresponds to a focal plane in the middle of the photoresist; (b,d) focal plane is at focus depth $d_{\mathrm{f}}=d_{\mathrm{f}, \mathrm{m}}$ in the middle of the photoresist of thickness $t_{\mathrm{PR}}$, (c,e) focus depth is shifted downward to value $d_{\mathrm{f}}<d_{\mathrm{f}, \mathrm{m}}$. Darker and lighter shades in photoresist in (b) to (d) indicate lower and higher effective exposure doses $D_{\exp }$, respectively; horizontal two-sided arrows in (b) and (c) indicate the sharp image of a dark structure of the projection reticle, projected within the photoresist with cones of opening angle $\theta_{1}$; (d) open and (e) buried microchannels obtained by the development of the photoresist in (b) and (c), respectively, after dissolution of photoresist of subcritical exposure $D_{\exp }<D_{\text {exp,cr. }}$. 
(a) Design 1

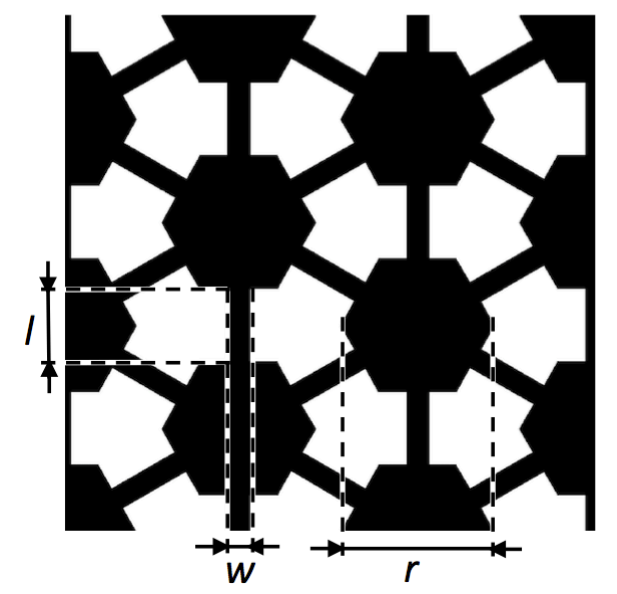

(b) Design 2

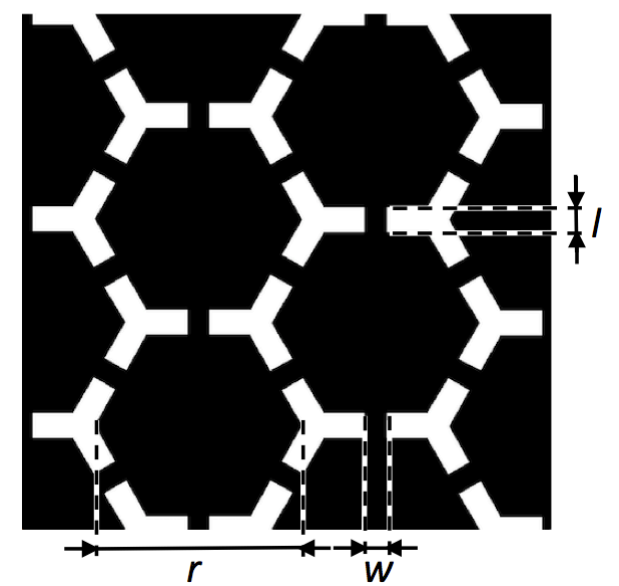

Figure 2. Schematic of the two designs of interconnected honeycomb structures on the projection reticle. Dark and white areas indicate opaque and transparent areas on the reticle, respectively. Geometrical dimensions of (a) Design 1: $l=150 \mu \mathrm{m}, w=50 \mu \mathrm{m}, r=200 \mu \mathrm{m}$; of (b) Design 2: $l=50 \mu \mathrm{m}, w=50 \mu \mathrm{m}, r=300 \mu \mathrm{m}$. All dimensions are shrunk by a factor of 5 by projection. 

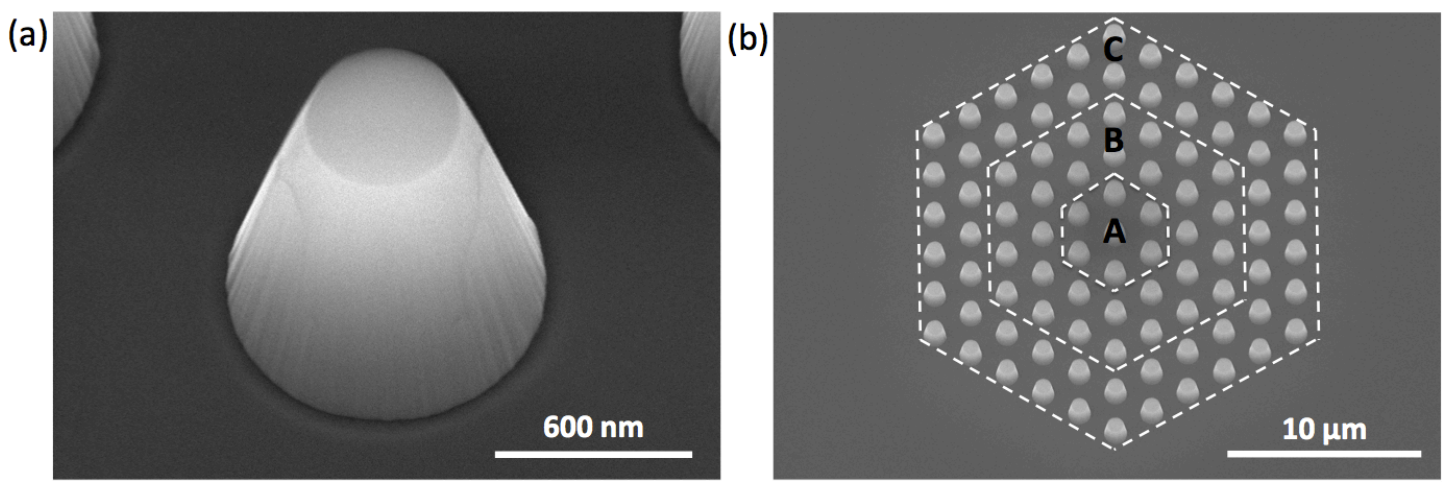

Figure 3. Scanning electron micrograph of (a) a nanopillar and (b) a hexagonal array of 91 nanopillars termed type C. Smaller arrays with 37 (type B) and 7 (type A) nanopillars, as outlined in (b), were realized as well. 
(a) Blocked channels

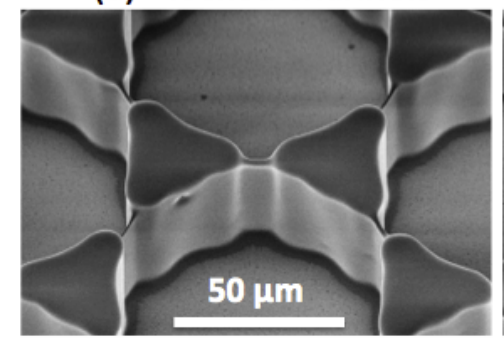

(b) Buried channels

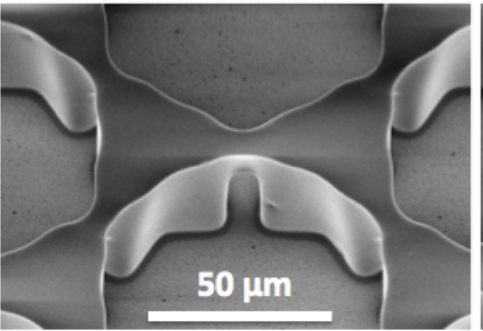

Figure 4. Scanning electron micrographs of cell container honeycombs of Design 2 obtained with different $d_{\mathrm{f}}$ values: containers (a) separated by thin walls, and connected by (b) buried and (c) (c) Surface channels

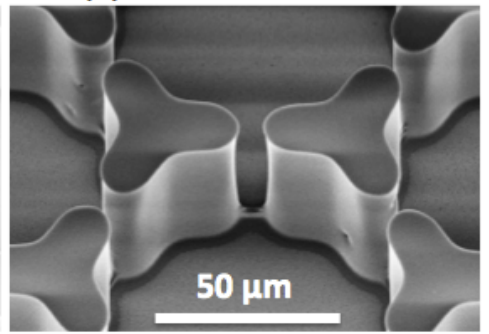

$$
\begin{aligned}
& \text { different } d_{\mathrm{f}} \text { values: containers (a) separated by thin walls, and } \\
& \text { surface microchannels. }
\end{aligned}
$$



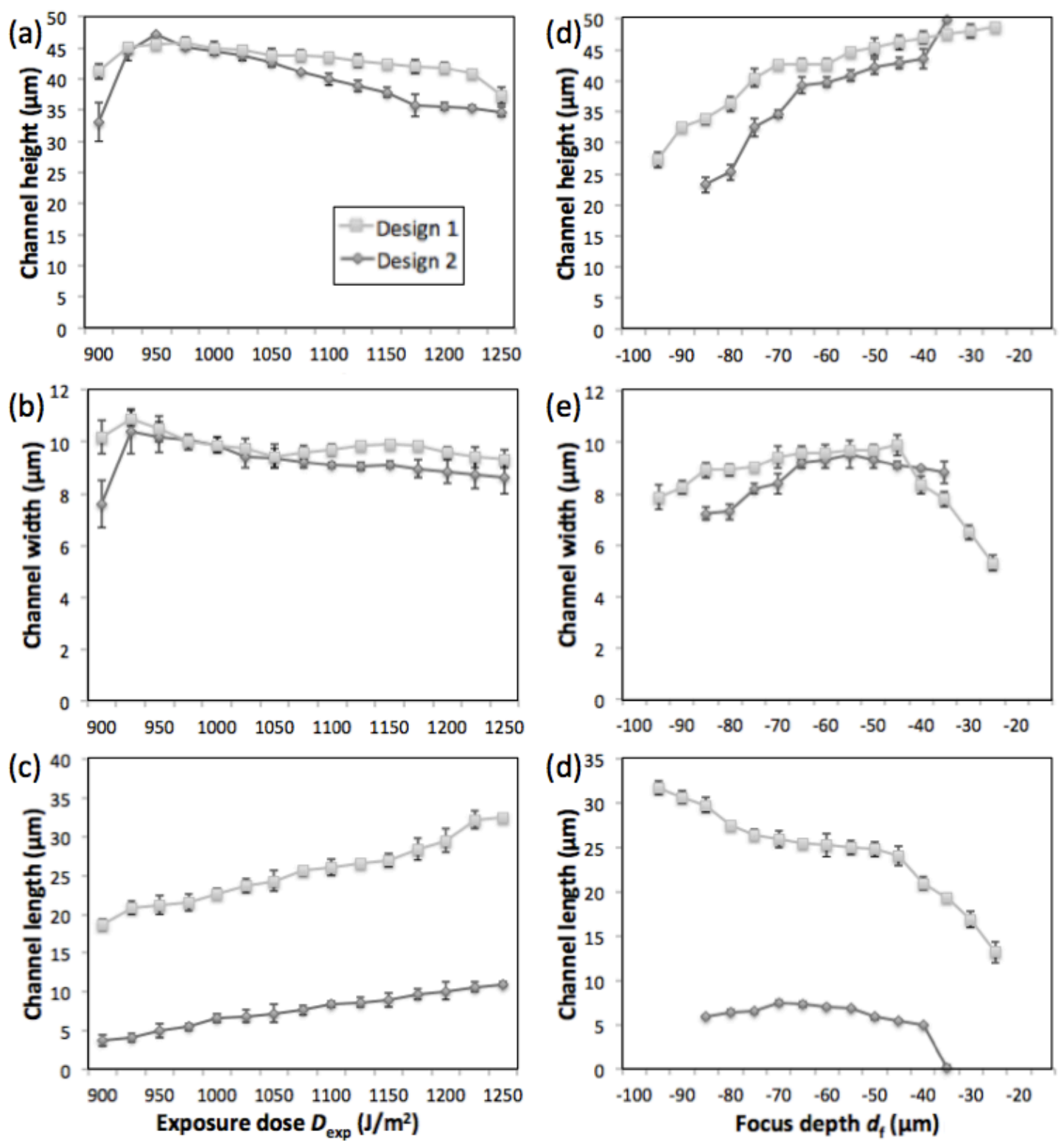

Figure 5. Measured height $(\mathrm{a}, \mathrm{d})$, width $(\mathrm{b}, \mathrm{e})$ and length $(\mathrm{c}, \mathrm{f})$ of microchannels as a function of (a,b,c) exposure dose $D_{\exp }$ for $d_{\mathrm{f}}=-50 \mu \mathrm{m}$ and (d,e,f) as a function of focus depth $d_{\mathrm{f}}$ for $D_{\exp }=1100 \mathrm{~J} / \mathrm{m}^{2}$ for Designs 1 (light grey symbols) and 2 (dark grey symbols) with $t_{\mathrm{PR}}=50 \mu \mathrm{m}$; plotted values represent averages and standard deviations from three wafers; lines are guides to the eye. 
(a)

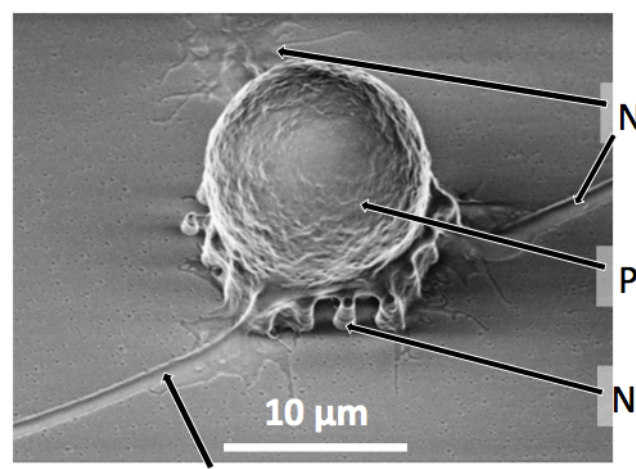

Neurites

(c)

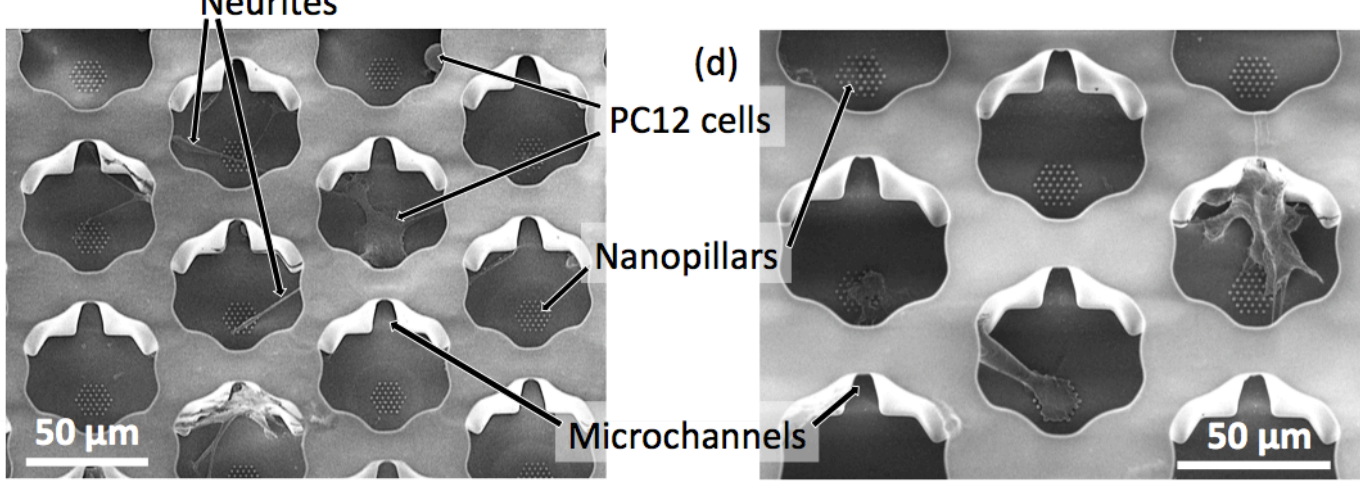

(b)

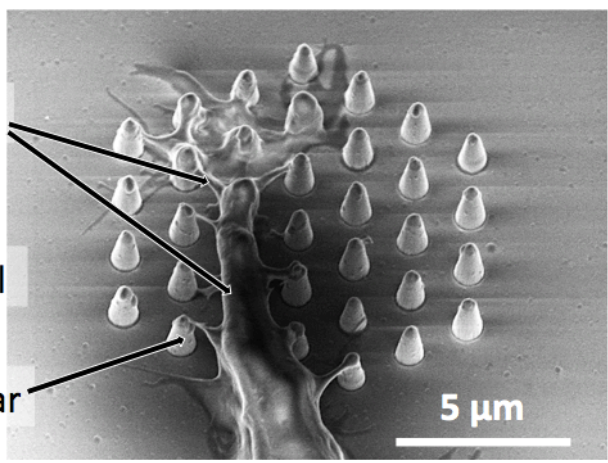

PC12 cell

Nanopillar

Figure 6. Scanning electron micrographs of (a) a neuron sitting on a nanopillar array with neurite growth, (b) a cell attached with several neurites to a nanopillar array, and (c,d) neurons prepositioned on nanopillar arrays by a Design 2 honeycomb arrangement of cell containers, with neurites extending across microchannels. All nanopillar arrays in (a) to (d) are of type B. 


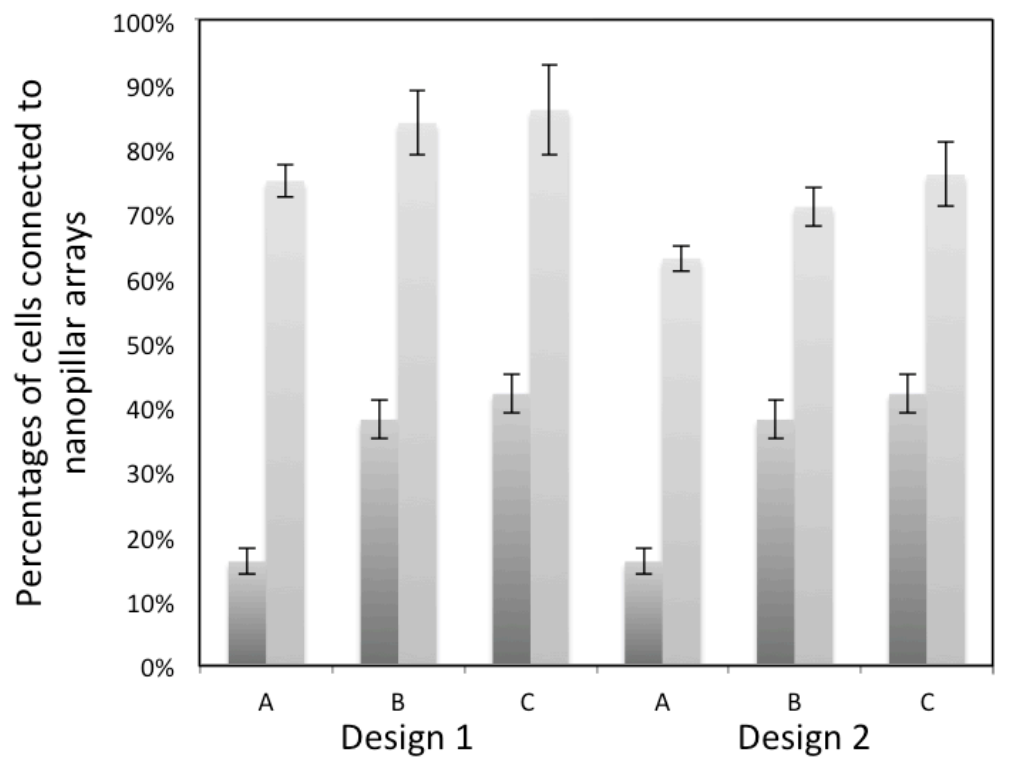

Figure 7. Percentages of cells connected to nanopillar arrays of types A, B, and C on silicon substrates without SU-8 structures (darker grey bars) and embedded in SU-8 interconnected honeycomb structures of Designs 1 and 2 (lighter grey bar). Values represent averages and standard deviations from 48 chips. 